Supporting Information

\title{
Efficient Photoexcited Charge Separation at the Interface of a Novel 0D/2D Heterojunction: A Time-Dependent Ultrafast Dynamic Study
}

\author{
Xueke $\mathrm{Yu},{ }^{\dagger}$ Yan $\mathrm{Su},{ }^{*}, \dagger$ Wen-wu $\mathrm{Xu},{ }^{\star}$ and Jijun $\mathrm{Zhao}^{\dagger}$ \\ ${ }^{\dagger}$ Key Laboratory of Materials Modification by Laser, Ion and Electron Beams (Dalian \\ University of Technology), Ministry of Education, Dalian 116024, China \\ ॠDepartment of Physics, School of Physical Science and Technology, Ningbo University, \\ Ningbo 315211, China
}




\section{S1. NAMD computational methods}

The time-dependent $a b$ initio nonadiabatic molecular dynamics (NAMD) was carried out with the Hefei-NAMD code ${ }^{1}$ to investigate the dynamics of excited carriers in $\mathrm{Au}_{9}\left(\mathrm{PH}_{3}\right)_{8} / \mathrm{MoS}_{2}$ heterostructure. After fully relaxing the initial structure at $0 \mathrm{~K}$, the $a b$ initio molecular dynamics (AIMD) $)^{2}$ simulation was performed to heat up the temperature of $\mathrm{Au}_{9}\left(\mathrm{PH}_{3}\right)_{8} / \mathrm{MoS}_{2}$ heterojunction to $300 \mathrm{~K}$, and the velocity scaling algorithm was adopted to achieve thermal equilibrium. Next, a 1 ps MD simulation was performed in the microcanonical ensemble with a $1 \mathrm{fs}$ atomic time-step. Due to a strong coupling between donor and acceptor states (see Table 1 and Figure 4a) and the quantum decoherence effect is weak enough to ignore, the photoelectron separation process (processes (1) in Figure 2a) was simulated by the fewest switches surface hopping $(\mathrm{FSSH})^{3,4}$ method. Decoherence induced surface hopping (DISH) ${ }^{5,6}$ method was adopted in the electron-hole recombination process (processes (3) in Figure 2a), since this process has a larger bandgap and significant quantum decoherence effect. For excited hole separation (processes (2) in Figure 2a), both the FSSH and DISH methods were adopted due to it has an energy gap of $0.4 \mathrm{eV}$. In all simulations, the Brillouin zones were sampled by $\Gamma$ point.

The population changes during $\mathrm{e}-\mathrm{h}$ recombination process is very small for both $\mathrm{MoS}_{2}$ sheet and $\mathrm{Au}_{9}\left(\mathrm{PH}_{3}\right)_{8} / \mathrm{MoS}_{2}$ heterojunction. To ensure the statistical reliability of the calculated population, an enormous number (5 000 000) of surface-hopping trajectories is adopted to calculate $\mathrm{e}-\mathrm{h}$ recombination with DISH method, which give a very small population change in the order of $2 \times 10^{-7}$ per time step. With a $1 \%$ uncertainly, the 1 ps NAMD run with the 1 fs nuclear integration time step can enable us to solve the recombination time scale around $0.01 \times 1 \times 5 \times 10^{9} \mathrm{fs}=50 \mathrm{~ns}$. To visually compare the e-h recombination processes of $\mathrm{MoS}_{2}$ sheet and $\mathrm{Au}_{9}\left(\mathrm{PH}_{3}\right)_{8} / \mathrm{MoS}_{2}$ heterojunction, we displayed the population evolution in the range of $0-2.75 \mathrm{~ns}$ (as shown in Figure 5a). Similarly, 100000 surface-hopping trajectories are adopted to calculate the dynamics of excited hole transfer with DISH method. 


\section{S2. Hole separation dynamics with DISH method}

As shown in Figure $\mathrm{S} 4 \mathrm{~b}$, the excited hole transfer is completed within 5 ps, which is about eight times longer than the result (680 fs) obtained by FSSH. The short puredephasing time ( $7.34 \mathrm{fs}$ ) between VBM and VBM-1 caused by the band gap is the main reason for the slower hole relaxation compared to the FSSH method (see Table S3). However, compared to the 205 ns required for electron-hole recombination, the hole relaxation process is still ultrafast. It can be explained by two reasons: i) The energy gap between VBM-1 to VBM is no more than $0.40 \mathrm{eV}$ with time increasing (see Figure $\mathrm{S} 4)$, which is less than $1.31 \mathrm{eV}$ for electron-hole recombination; ii) The pure-dephasing time for hole transfer (7.34 fs) is close to electron-hole recombination (7.4 fs), while the NAC between adjacent states $(>14.7 \mathrm{meV})$ is notably larger than that of recombination process $(0.14 \mathrm{meV})$ (see Tables 1 and $\mathrm{S} 2)$. 
Table S1. Calculated average bond distances $(\AA)$ for ligand protected clusters $\mathrm{Au}_{9}\left(\mathrm{PH}_{3}\right)_{8}$ and $\mathrm{Au}_{9}\left(\mathrm{PPh}_{3}\right)_{8}$ (see Figure $\mathrm{S} 1$ ) in comparison with experimental geometrical parameters for $\mathrm{Au}_{9}\left(\mathrm{PPh}_{3}\right)_{8}$.

\begin{tabular}{lcccc}
\hline \hline & Bonds & $\mathrm{Au}_{9}\left(\mathrm{PH}_{3}\right)_{8}$ & $\mathrm{Au}_{9}\left(\mathrm{PPh}_{3}\right)_{8}$ & Exp. $^{7}$ \\
\hline & $1-2,1-3,1-4,1-5$ & 2.69 & 2.68 & 2.68 \\
& $1-6,1-7,1-8,1-9$ & 2.98 & 2.97 & 2.71 \\
$\mathrm{Au}-\mathrm{Au}$ & $2-3,4-5$ & 2.79 & 2.79 & 2.77 \\
& $6-7,8-9$ & 2.66 & 2.66 & 2.76 \\
& $2-8,2-9,3-6,3-7$, & & & 2.89 \\
$\mathrm{Au}-\mathrm{P}$ & $4-8,4-9,5-6,5-7$ & 2.95 & 2.94 & \\
\hline \hline
\end{tabular}


Table S2. Nonadiabatic coupling (NAC) along 1 ps NAMD trajectory between the donor and acceptor states for hole relaxation of $\mathrm{Au}_{9}\left(\mathrm{PH}_{3}\right)_{8} / \mathrm{MoS}_{2}$ heterojunction.

\begin{tabular}{ccccc}
\hline \hline NAC (meV) & VBM-3 & VBM-2 & VBM-1 & VBM \\
\hline VBM-3 & 0.00 & 26.09 & 4.50 & 2.16 \\
VBM-2 & 26.09 & 0.00 & 47.24 & 0.82 \\
VBM-1 & 4.50 & 47.24 & 0.00 & 14.70 \\
VBM & 2.16 & 0.82 & 14.70 & 0.00 \\
\hline \hline
\end{tabular}


Table S3. Pure-dephasing $(\tau)$ along 1 ps NAMD trajectory between the donor and acceptor states for hole relaxation of $\mathrm{Au}_{9}\left(\mathrm{PH}_{3}\right)_{8} / \mathrm{MoS}_{2}$ heterojunction.

\begin{tabular}{ccccc}
\hline \hline$\tau(\mathrm{fs})$ & VBM-3 & VBM-2 & VBM-1 & VBM \\
\hline VBM-3 & 0.00 & 60.31 & 42.63 & 7.04 \\
VBM-2 & 60.31 & 0.00 & 911.41 & 7.21 \\
VBM-1 & 42.63 & 911.41 & 0.00 & 7.34 \\
VBM & 7.04 & 7.21 & 7.34 & 0.00 \\
\hline \hline
\end{tabular}



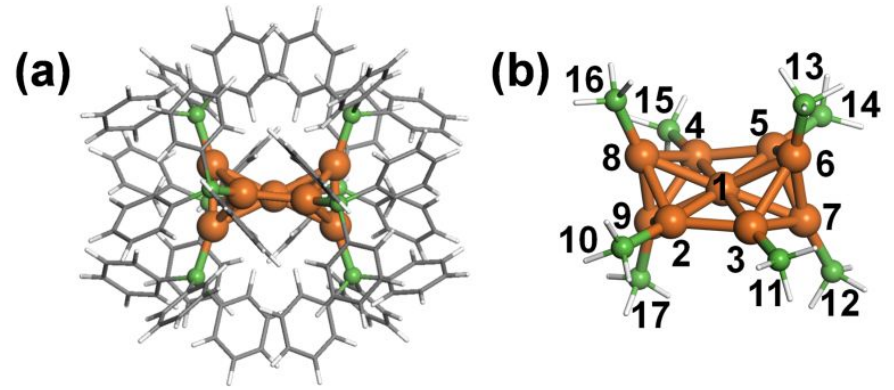

Figure S1. Atomic structures of (a) $\mathrm{Au}_{9}\left(\mathrm{PPh}_{3}\right)_{8}$ and (b) $\mathrm{Au}_{9}\left(\mathrm{PH}_{3}\right)_{8}$ with atomic number. Orange, green, gray, and white balls represent $\mathrm{Au}, \mathrm{P}, \mathrm{C}$, and $\mathrm{H}$ atoms, respectively. 

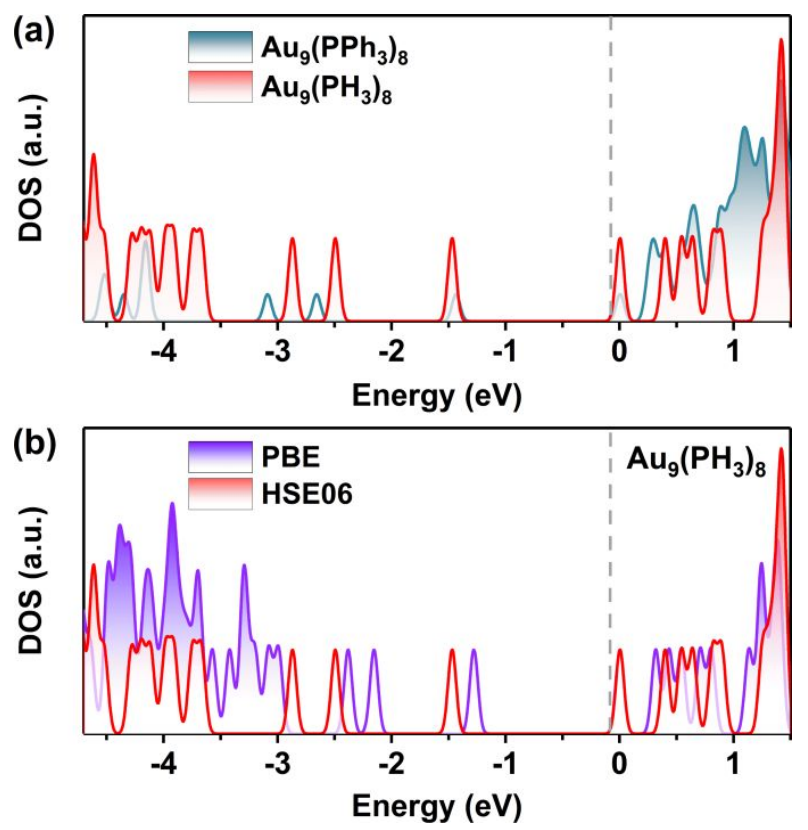

Figure S2. (a) Density of states of $\mathrm{Au}_{9}\left(\mathrm{PPh}_{3}\right)_{8}$ and $\mathrm{Au}_{9}\left(\mathrm{PH}_{3}\right)_{8}$ calculated using HSE06 functionals. (b) Density of states of $\mathrm{Au}_{9}\left(\mathrm{PH}_{3}\right)_{8}$ calculated using HSE06 and PBE functionals. The dashed gray lines indicate the Fermi level, which is set to $0 \mathrm{eV}$. 


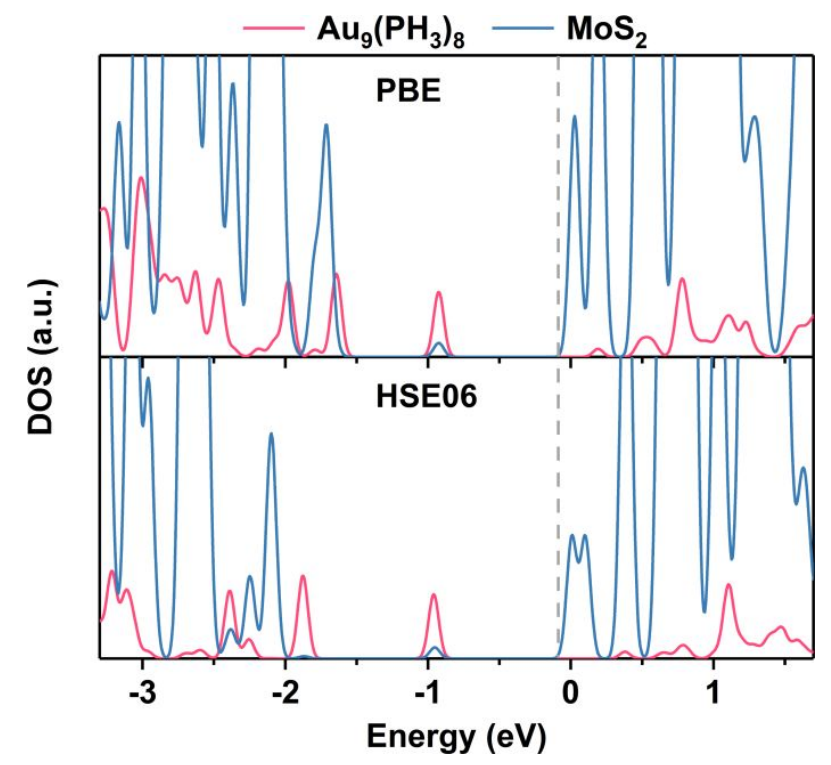

Figure S3. Density of states of $\mathrm{Au}_{9}\left(\mathrm{PPh}_{3}\right)_{8} / \mathrm{MoS}_{2}$ heterojunction calculated by $\mathrm{PBE}$ (top panel) and HSE06 (bottom panel) functionals. The dashed gray line indicates the Fermi level, which is set to 0 $\mathrm{eV}$. 

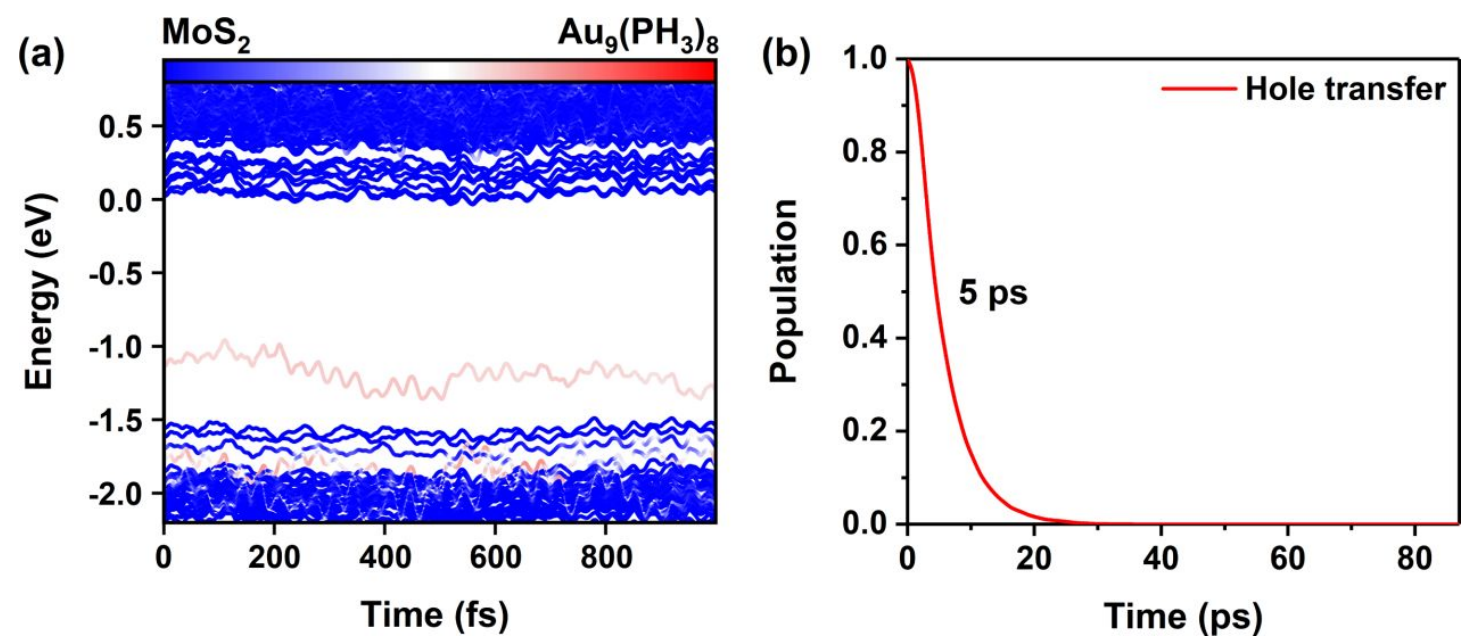

Figure S4. (a) Time-dependent energy states evolution of $\mathrm{Au}_{9}\left(\mathrm{PH}_{3}\right)_{8} / \mathrm{MoS}_{2}$ heterojunction. The color map indicates the state distribution on $\mathrm{Au}_{9}\left(\mathrm{PH}_{3}\right)_{8}$ and $\mathrm{MoS}_{2}$. (b) Population evolution of the excited hole during hole separation process for $\mathrm{Au}_{9}\left(\mathrm{PH}_{3}\right)_{8} / \mathrm{MoS}_{2}$ heterojunction with DISH method. 


\section{References}

(1) Zheng, Q.; Chu, W.; Zhao, C.; Zhang, L.; Guo, H.; Wang, Y.; Jiang, X.; Zhao, J. Ab Initio Nonadiabatic Molecular Dynamics Investigations on the Excited Carriers in Condensed Matter Systems. WIREs Comput. Mol. Sci. 2019, 9, e1411.

(2) Kresse, G.; Hafner, J. Ab Initio Molecular Dynamics for Liquid Metals. Phys. Rev. B 1993, $47,558-561$

(3) Craig, C. F.; Duncan, W. R.; Prezhdo, O. V. Trajectory Surface Hopping in the TimeDependent Kohn-Sham Approach for Electron-Nuclear Dynamics. Phys. Rev. Lett. 2005, 95, 163001 .

(4) Tully, J. C. Molecular Dynamics with Electronic Transitions. J. Chem. Phys. 1990, 93, 10611071.

(5) Jaeger, H. M.; Fischer, S.; Prezhdo, O. V. Decoherence-induced Surface Hopping. J. Chem. Phys. 2012, 137, 22A545.

(6) Trivedi, D. J.; Prezhdo, O. V. Decoherence Allows Model Reduction in Nonadiabatic Dynamics Simulations. J. Phys. Chem. A 2015, 119, 8846-8853.

(7) Wen, F.; Englert, U.; Gutrath, B.; Simon, U. Crystal Structure, Electrochemical and Optical Properties of $\left[\mathrm{Au}_{9}\left(\mathrm{PPh}_{3}\right)_{8}\right]\left(\mathrm{NO}_{3}\right)_{3}$. Eur. J. Inorg. Chem. 2008, 2008, 106-111. 